\title{
TRATAMIENTO QUIRURGICO DEL PROLAPSO GENITAL Y SU CONTROL INMEDIATO
}

\author{
Dr. Antonio Vélez Zapata - Dr. Dagoberto Gaviria Suárez
}

Hospital Universitario San Vicente de Paúl - Medellín, Colombia

El presente estudio se realizó en el Departamento de Ginecología y Obstetricia del Hospital Universitario San Vicente de Paúl, utilizando el Archivo de Historias Clínicas de pacientes intervenidas quirúrgicamente para corrección de Prolapso Genital, en un período de cinco años, comprendido entre el $1^{\circ}$ de Enero de 1960 y el 31 de Diciembre de 1964.

El total de las pacientes aceptadas en los Servicios Generales del Hospital en este período de observación, se elevó a 123.946, de los cuales 3.659 lo fueron en el Servicio de Ginecología. Entre ellas 2.425 necesitaron tratamiento quirúrgico por ginecopatía. De ellas 478 lo fueron por prolapso genital, lo cual representa el $13.7 \%$ de las enfermas internadas y muy cerca del $20 \%$ del total de las intervenciones quirúrgicas ginecológicas.

Edad. En relación con la edad de las pacientes tenemos lo siguiente:

La paciente de mayor edad alcanzó a los 85 años, sin que esto fuera considerado como una contraindicación quirúrgica, por sí sola. La paciente más joven tenía 19 años y ya

\section{CUADRO NN 1 \\ DISTRIBUCION POR EDADES}

\begin{tabular}{lrrr}
\hline Edad en años & No de Casos & $\%$ \\
\hline $\begin{array}{l}\text { Menores de } 20 \\
21\end{array}$ a años 30 & 1 & 0.2 \\
31 a 40 & 30 & 6.2 \\
41 a 50 & 99 & 20.5 \\
51 a 60 & 141 & 29.7 \\
61 a 70 & 120 & 25.2 \\
Más de 71 & 61 & 12.8 \\
TOTAL: & 26 & 5.4 \\
\hline
\end{tabular}

había llevado a término 4 embarazos, en el momento de acudir al tratamiento quirúrgico de su prolapso. El mayor porcentaje puede observarse entre los 31 y los 60 años, con un $75.4 \%$.

Paridad. Se contabilizaron en total 4.188 partos, de los cuales solamente 52 fueron atendidos por médico. El resto 4.136 fueron atendidos por comadronas. Es necesario explicar que la inmensa mayoría de las pacientes, provienen de áreas rurales, en las cuales se carece de atención médica. Otras causas que ayudan a explicar este hecho, lo son: el nivel cultural y las condiciones socio-económicas de nuestras pacientes hospitalarias. 
CUADRO N: 2

\section{RELACION DE LA PARIDAD} $Y$ PROLAPSO GENITAL

\begin{tabular}{|c|c|c|c|}
\hline \multicolumn{2}{|l|}{ Paridad } & $\mathrm{N}^{\circ}$ de Casos & $\%$ \\
\hline \multicolumn{2}{|l|}{ Nulíparas } & 6 & 1.2 \\
\hline $1 \quad a$ & 3 & 93 & 19.3 \\
\hline 4 & 6 & 103 & 21.9 \\
\hline $7 \quad a$ & 10 & 132 & 27.9 \\
\hline 11 a & 14 & 91 & 18.5 \\
\hline 15 a & 18 & 46 & 9.8 \\
\hline Más de & 18 & 7 & 1.4 \\
\hline \multicolumn{2}{|l|}{ TOTAL : } & 478 & $100 \%$ \\
\hline
\end{tabular}

Seis de las pacientes eran nulíparas. La de mayor paridad, alcanzó 24 partos a término. La mayor incidencia la presentó el grupo de 4 a 10 partos.

Fueron intervenidos, en el grupo de los atendidos por médico, 12 casos por fórceps, una operación cesárea y 6 extracciones podálicas.

\section{GRADO CLINICO DEL PROLAPSO}

Se hizo la clasificación, de acuerdo a las recomendaciones internacionales, en grados I, II y III, valorando el descenso uterino, para los prolapsos totales, y de la víscera descendida (Vagina, vejiga, recto, intestino) para los prolapsos parciales.

\section{Grado Clínico del Prolapso Total}

Análisis de 425 intervenidas en el Hospital Universitario San Vicente de Paúl. Enero de 1960 Diciembre de 1964. (Ver Cuadro № 3).

\section{CUADRO No 3}

GRADO CLINICO DEL PROLAPSO TOTAL

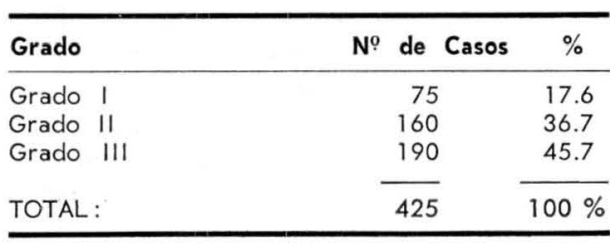

En los prolapsos parciales, únicamente se consideraron quirúrgicos grados II y III, como se explica en el cuadro siguiente.

\section{Grado Clínico de los Prolapsos Parciales}

En 56 casos intervenidos en el Hospital Universitario San Vicente de Paúl. Enero de 1960. Diciembre de 1964. (Ver Cuadro № 4)

\section{CUADRO N: 4}

GRADO CLINICO DE LOS PROLAPSOS PARCIALES

\begin{tabular}{|c|c|c|c|}
\hline Tipo & Grado II & Grado III & Total \\
\hline Cistocele puro & 2 & 6 & 8 \\
\hline Cisto y Rectocele & 16 & 19 & 35 \\
\hline Rectocele puro & 2 & 6 & 8 \\
\hline Prolapso de cúpula & - & - & 5 \\
\hline TOTAL : & & & 56 \\
\hline
\end{tabular}

Asociación del Prolapso a otras entidades ginecológicas

(Ver Cuadro № 5).

\section{CUADRO N: 5}

ASOCIACION DEL PROLAPSO A OTRAS ENTIDADES GINECOLOGICAS

\begin{tabular}{llr}
\hline & Casos \\
\hline Prolapso e Incontinencia Urinaria de & \\
esfuerzo & 39 \\
Prolapso y desgarro perineal III & 11 \\
Prolapso y Ca. Invasor de Cuello Uterino & 1 \\
Prolapso y Ca. in situ de Cuello Uterino & 5 \\
Prolapso y enterocele & 8 \\
Prolapso y quiste de lovario & 3 \\
Prolapso y Pólipos cervicales & 2 \\
Prolapso y Mioma Uterino & 3 \\
Prolapso y Fístula Uretrovaginal & 1 \\
Prolapso y Leucoplasia vulvar & 1 \\
& & -14 \\
TOTAL:
\end{tabular}

\section{ESTUDIOS CITOLOGICOS PARA CARCINOMA}

En nuestro servicio, se ha establecido como requisito indispensable, 
para obtener el $V: B$ ? del revisor quirúrgico, el descartar por medio de los estudios citológicos, la presencia de neoplasia maligna en el tracto genital. Cuando los frotis resultan sospechosos o positivos el diagnóstico se confirma por medio del estudio Anatomopatológico de la biopsia.

Se hicieron 436 estudios citológicos, los cuales se calificaron de acuerdo a los principios de Papanicolaou, así: (Ver Cuadro № 6).

\section{CUADRO NN 6}

ESTUDIOS CITOLOGICOS

\begin{tabular}{|c|c|c|c|c|c|}
\hline Grado & Citológico & $\mathbf{N}^{\circ}$ & de & Casos & $\%$ \\
\hline & 1 & & 37 & & 8.4 \\
\hline & 11 & & 356 & & 81.6 \\
\hline & III & & 33 & & 7.5 \\
\hline & IV & & 4 & & 1.0 \\
\hline & V & & 6 & & 1.5 \\
\hline \multicolumn{2}{|c|}{ TOTAL: } & & 436 & & $100 \%$ \\
\hline
\end{tabular}

Las pacientes con extendidos citológicos grado 111 , fueron tratadas $y$ controladas posteriormente. Solamente persistieron como tales tres de ellos, en los cuales se practicó biopsia por sacabocado y estudio anatomopatológico. Los tres informes fueron similares, indicando la presencia de Cervicitis crónica y metaplasia escamosa.

Todos los frotis calificados como grado IV, fueron seguidos de biopsia cervical y los resultados fueron: 3 ćasos de metaplasia escamosa y 1 carcinoma intraepitelial.

El Papanicolaou informado como grado $V$, correspondió en el estudio anatomopatológico: Carcinoma intraepitelial en 4 casos. Carcinoma invasor en 1 caso y metaplasia escamosa con atipias, en otro caso.

\section{BIOPSIAS DEL CUELLO UTERINO}

Se realizaron 32 biopsias de cuello previas a la consideración del revisor quirúrgico. Algunas de ellas en pacientes en las cuales no fué posible realizar el estudio citológico por dificultades técnicas en el laboratorio - ante la más mínima sospecha en un cuello lesionado. Los resultados fueron:

1 Carcinoma Invasor.

5 Carcinomas in situ.

1 Metaplasia escamosa con atipias.

25 Cervicitis y metaplasia escamosa.

\section{BIOPSIAS DE ENDOMETRIO}

En pacientes con metrorragias $O$ trastornos del ciclo menstrual, se hacía imperiosa la biopsia de endometrio con el fin de descartar patología intrauterina. Se practicaron un total de 15 biopsias de endometrio, cuyos resultados se consignan a continuación:

$\begin{array}{lr}\text { Endometrio proliferativo } & 10 \text { casos } \\ \text { Endometrio secretor } & 2 \text { casos } \\ \text { Endometritis } & 2 \text { casos } \\ \text { Decidua } & 1 \text { caso }\end{array}$

Técnicas quirúrgicas. Se intervinieron el total de las 478 enfermas consideradas en este estudio. Unicamente se rechazaron dos pacientes, en las cuales se confirmó la presencia de un embarazo inicial, logrado en el lapso transcurrido entre el $\mathrm{V}: \mathrm{B}$ ? quirúrgico y la admisión al Hospital. Las técnicas quirúrgicas empleadan fueron: (Ver Cuadro No 7).

Elección de la técnica. En la decisión final respecto a la técnica adecuada para cada uno de los casos presentados al Revisor Quirúrgico se tuvieron en cuenta los siguientes factores: Edad de la paciente, grado del prolapso, tamaño del útero, porvenir obstétrico, vida marital y la asociación del prolapso a otras ginecopatías. 
CUADRO N 7

TECNICAS QUIRURGICAS

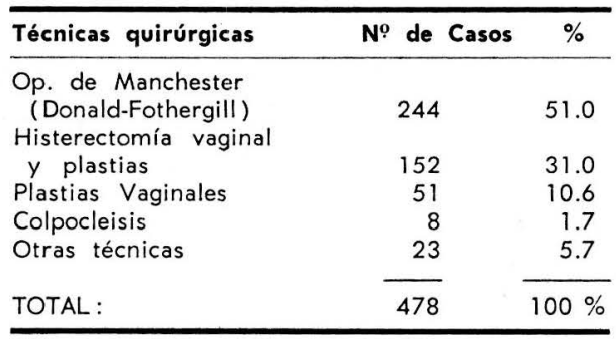

Las plastias vaginales anterior $y$ posterior, con perineorrafia, se realizaron en mujeres jóvenes que presentaban cisto y rectocele, lo mismo que algún grado de desgarro perineal y en las que el histerocele no existía o era apenas incipiente.

Las plastias vaginales combinadas con histerofijaciones o ligamentopexias, en las pacientes jóvenes que presentaban prolapso integral, y cuyo porvenir obstétrico era de primerísima importancia.

La operación de Le Fort se practicó en pacientes de avanzada edad, que presentaban prolapso de II y III grado y en las cuales se había descartado otra patología ginecológica agregada.
La histerectomía vaginal asociada a las plastias vaginales complementarias, se realizó en las pacientes con prolapso genital integral de tercer grado, cuando se encontraba patología ginecológica asociada o cuando por el grado de la hernia pélvica se consideraba más ventajoso la extirpación del útero, con fines a corregir el prolapso, claro está que en estas pacientes no era de considerarse ya el porvenir obstétrico.

La técnica de Manchester (DonaldFothergill*), goza en nuestro Servicio de Ginecología de la mayor amplitud, en cuanto a indicación para corrección del prolapso genital, no importando el grado del mismo, ni la edad de la paciente, considerando sí el porvenir obstétrico de las mismas para ser muy cuidadosos en la amputación moderada del cuello uterino. Las demás técnicas son efectuadas muchas veces con fines docentes pero con indicaciones muy precisas.

\section{TIPOS DE INTERVENCIONES. PROLAPSO INTEGRAL}

Análisis de 425 casos en el Hospital Universitario San Vicente de Paúl de 1960 a 1964. (Ver Cuadro No 8).

\section{CUADRO № 8}

TIPOS DE INTERVENCIONES - PROLAPSO GENITAL

\begin{tabular}{|c|c|c|c|c|c|}
\hline Operación & Grado I & Grado II & Grado III & Total & $\%$ \\
\hline Mánchester & 44 & 91 & 90 & 225 & 53.5 \\
\hline Histerectomía y Plastias & & 59 & 91 & 150 & 33.6 \\
\hline Plastias Anterior y Posterior & 11 & 5 & & 16 & 4.0 \\
\hline Mánchester-Kelly & 3 & 4 & 4 & 11 & 2.9 \\
\hline Fijación y Plastias & & 3 & 7 & 10 & 2.5 \\
\hline Le Fort & 1 & 3 & 3 & 7 & 1.8 \\
\hline Plastias-Kelly & 2 & 1 & & 3 & 0.8 \\
\hline Amp. cuello y Perineorrafia & & & 2 & 2 & 0.6 \\
\hline Halban & & 1 & & 1 & 0.3 \\
\hline TOTALES : & 61 & 167 & 197 & 425 & $100 \%$ \\
\hline
\end{tabular}




\section{TIPOS DE INTERVENCIONES EN LOS PROLAPSOS PARCIALES}

En los prolapsos parciales se acomodó la técnica al prolapso, según su magnitud y el tipo de órgano descendido. La colpoperineorrafia obligatoria en todos los casos.

Análisis de 56 casos en el Hospital Universitario San Vicente de Paúl, de 1960 a 1964. (Ver Cuadro № 9).

\section{CUADRO N: 9}

INTERVENCIONES QUIRURGICAS

EN LOS PROLAPSOS PARCIALES

\begin{tabular}{lcc}
\hline Técnica & $N^{0}$ de Casos & $\%$ \\
\hline $\begin{array}{l}\text { Plastias anterior y posterior } \\
\text { Manchester }\end{array}$ & 35 & 66.2 \\
$\quad$ (Donald-Fothergill) & 8 & 15.2 \\
Histerectomía Vaginal & 2 & 6.2 \\
y Plastias & 1 & 3.1 \\
Colpocleisis & 1 & 3.1 \\
Colpoperineorrafia & 1 & 3.1 \\
Colpectomía parcial & 1 & 3.1 \\
Operación de Kelly & 56 & $100 \%$ \\
TOTALES: & & \\
\hline
\end{tabular}

Prolapso de cúpula vaginal. Se contabilizaron 6 casos de prolapso de cúpula vaginal que se habían producido, años después de una histerectomía vaginal para corrección de prolapso genital. El tratamiento en estos casos fué:

Colpocleisis parcial 5 casos Fijación (vía abdominal) 1 caso Técnica de Williams-Richardson.

Reproducciones del prolapso. Se reintervinieron tres pacientes, que habían sufrido cirugías previas para corrección de su prolapso genital y que se pueden detallar así:

Dos reproducidas después de una operación de Mánchester y una después de una operación de Halban.

Influencia de la edad en el tipo de intervención. Los promedios de edad para las pacientes de acuerdo a la técnica quirúrgica empleada fue: (Ver Cuadro № 10).

\section{CUADRO NN 10}

INFLUENCIA DE LA EDAD EN EL TIPO DE INTERVENCION QUIRURGICA

$\begin{array}{llll}27 & \text { años } & 22-44 & \text { años } \\ 45 & \text { " } & 19-70 & \text { "' } \\ 47 & 24-85 & \text { " } & 32-74 \\ 52 & \text { " } & 32-80 & \text { "' } \\ 78 \text { " } & 62-80\end{array}$

Se considera una paciente de apenas 32 años, quien se sometió a una histerectomía total y plastias complementarias. Es necesario advertir que se trataba de una verdadera hernia pélvica y había cumplido ya con su función procreadora, con 4 hijos a término.
Frecuencia de las complicaciones en relación con el tipo de intervención

Análisis en 478 pacientes con Prolapso genital en el Hospital Universitario San Vicente de Paúl de Enero de 1960 a Diciembre de 1964. (Ver Cuadro № 11 ). 
CUADRO N: 11

FRECUENCIA DE LAS COMPLICACIONES EN RELACION CON EL TIPO DE INTERVENCION

\begin{tabular}{|c|c|c|c|c|c|}
\hline \multirow[b]{2}{*}{ Número } & \multirow{2}{*}{$\begin{array}{c}\begin{array}{c}\text { Operación de } \\
\text { Manchester }\end{array} \\
244\end{array}$} & \multirow{2}{*}{$\begin{array}{c}\begin{array}{l}\text { Histerectomía } \\
\text { Vag. y Plastias }\end{array} \\
152\end{array}$} & \multirow{2}{*}{$\begin{array}{c}\text { Plastias } \\
\text { Vagina } \\
51\end{array}$} & \multicolumn{2}{|c|}{$\begin{array}{c}\text { Cleisis } \\
\text { Total }\end{array}$} \\
\hline & & & & 8 & $\%$ \\
\hline Infecciones Urinarias & $14-5,7 \%$ & $20-13,1 \%$ & $4-7,8 \%$ & 0 & 26 \\
\hline Hemorragia Intraoperatoria & $12-4,9 \%$ & $11-7,2 \%$ & $2-3,9 \%$ & 1 & 12,5 \\
\hline Hemorragia Postoperatoria & $11-4,5 \%$ & $5-3,3 \%$ & $1-1,9 \%$ & 0 & \\
\hline Absceso de Vagina & $7-2,9 \%$ & $13-8,5 \%$ & 0 & 2 & $25 \%$ \\
\hline Retención Urinaria & $7-2,9 \%$ & $9-5,9 \%$ & 0 & 0 & \\
\hline Hematomas & $2-0,8 \%$ & $3-2,0 \%$ & 0 & 0 & \\
\hline Dehiscencia de Pared & $1-0,4 \%$ & $3-2,0 \%$ & $1-1,9 \%$ & 0 & \\
\hline Ruptura Vesical & $0-$ & $0-$ & $1-1,9 \%$ & 0 & \\
\hline Ruptura de Uretra & $1-0,4 \%$ & 0 & 0 & 0 & \\
\hline Absceso Pélvico & $0-$ & $3-2,0 \%$ & 0 & 0 & \\
\hline Absceso de Cúpula & 0 & $5-3,3 \%$ & 0 & 0 & \\
\hline
\end{tabular}

El mayor porcentaje de complicación con la histerectomía vaginal, está probablemente relacionado con el mayor traumatismo tisular, la mayor pérdida sanguínea y la duración del acto quirúrgico. La infección urinaria ocupó el primer puesto de las complicaciones con un total de 38 casos $26.6 \%$.

Hay cuatro causas principales de la infección urinaria en el postoperatorio que son: la cateterización, la presencia de orina residual, el traumatismo operatorio y la anemia.

\section{Revisiones post-quirúrgicas}

En el momento de dar de alta a la paciente se le instruye adecuadamente respecto a concurrir a la Consulta Externa de Ginecología, para revisión postoperatoria, en el término de las 6 a las 8 semanas después de su salida del Hospital.

A pesar del interés de los médicos en este aspecto, únicamente se logró que asistieran al control 225 de las 478 pacientes intervenidas, lo cual representa el $47.3 \%$. En el término de los tres primeros meses se presentaron 195 pacientes y después de este lapso, 30 pacientes. Estudiamos las secuelas y recidivas en este grupo de pacientes.

Secuelas. Constituyeron las secuelas más frecuentes en el grupo de 225 pacientes de las 478 intervenidas. $(47.5 \%)$ que asistieron siquiera una vez a la revisión postoperatoria, las que enumeramos a continuación: (Ver Cuadro N․ 12).

\section{CUADRO NN 12}

\section{SECUELAS QUIRURGICAS EN 225 CASOS PROLAPSO GENITAL}

\begin{tabular}{lcc}
\hline Secuelas & $N^{0}$ de Casos & $\%$ \\
\hline Estrechez Vaginal y & & \\
Dispareunia & 17 & 7.5 \\
Incontinencia Urinaria & 12 & 5.3 \\
de esfuerzo & 7 & 3,1 \\
Infecciones Urinarias & 2 & 0.9 \\
Disuria Persistente & 2 & 0,9 \\
Acortamiento Vaginal & 1 & 0.4 \\
Infección Vaginal & 1 & 0.4 \\
Retención Urinaria Crónica & 1 & 0.4 \\
Bridas Vaginales & 1 & 0.4 \\
Fístulas Uretrales & 1 & 0.4 \\
Dehiscencia de pared vaginal & 1 & 0.4 \\
Hematoma infectado & & \\
\hline
\end{tabular}

Recidivas. En las revisiones postoperatorias se pudo comprobar la presencia de los siguientes prolapsos reproducidos: 


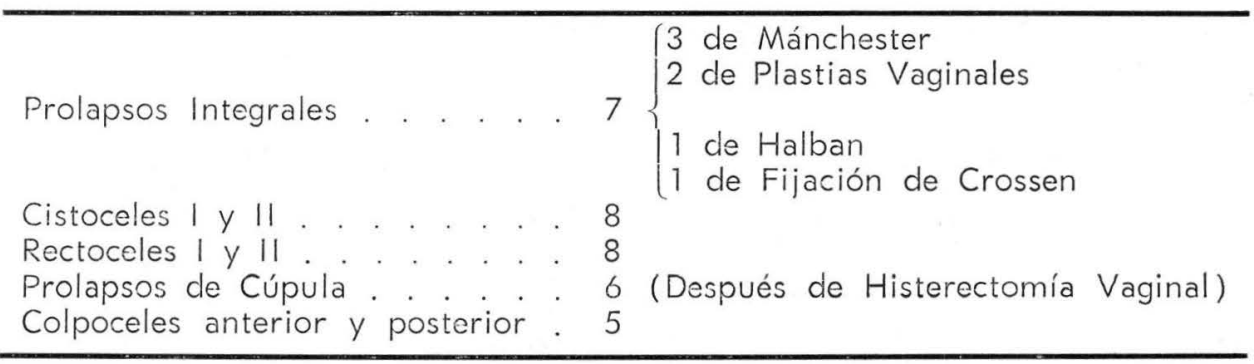

Anostesia. Estuvo encomendada al personal docente $y$ en entrenamiento del Departamento de Anestesiología. Son ellos los encargados del control pre-anestésico y de la elección de la anestesia más indicada a cada paciente en particular. Se utilizó en el curso de las intervenciones. (Ver Cuadro No 13).

\section{CUADRO N: 13}

ANESTESIA UTILIZADA EN LAS INTERVENCIONES DEL PROLAPSO GENITAL

\begin{tabular}{|c|c|c|c|}
\hline Anostesia & Nọ de & Casos & $\%$ \\
\hline General & 318 & & 66.53 \\
\hline Raquídea & 124 & & 25.93 \\
\hline Epidural & 30 & & 6.28 \\
\hline Combinada & 6 & & 1.26 \\
\hline TOTAL : & 478 & & $100 \%$ \\
\hline
\end{tabular}

El pequeño grupo de las anestesias combinadas, se explica ante el fracaso, con una de las técnicas de bloqueo, o cuando por prolongarse el acto quirúrgico exigía la anestesia general complementaria.

Anaromía patológica. Se tiene como práctica de rutina en los servicios quirúrgicos del Hospital, enviar para estudio histológico todos los especímenes quirúrgicos para su análisis cuidadoso. No se exceptuaban a este principio los cuellos extirpados con una oparación de Mánchester o los úteros obtenidos por histerectomía, aunque no existiesen signos sospechosos de malignidad.
En 60 de las intervenciones, no se obtuvo ningún material para estudio histológico. En 27 de las historias, no se encontró informe del estudio de la pieza quirúrgica enviada. (Ver Cuadro N: 14).

\section{CUADRO № 14}

ESTUDIO HISTOLOGICO DE LOS CUELLOS UTERINOS EXTIRPADOS

\begin{tabular}{lrc}
\hline Entidad & No de Casos & $\%$ \\
\hline Cervicitis crónicas & 168 & 42.2 \\
Cervicitis crónicas y & 154 & 38.7 \\
Metaplasia escamosa & 54 & 13.7 \\
Metaplasia escamosa & 6 & 1.6 \\
Cervicitis aguda & 5 & 1.3 \\
Carcinoma "in situ" & 5 & 1.3 \\
Endocervicitis & 2 & 0.6 \\
Pólipos Endocervicales & 2 & 0.6 \\
Cuello normal & 399 & $100 \%$ \\
& & 39 \\
\hline
\end{tabular}

Como puede apreciarse, la inmensa mayoría de los cuellos uterinos estudiados presentaron cervicitis crónica y/o cervicitis asociada a metaplasia escamosa. La coexistencia de Prolapso y Carcinoma "in situ", se presentó en 5 casos. (1,3\%).

\section{Estudio histológico de los úteros extirpados}

(Cuadro No 15).

El útero senil, encontrado en el mayor porcentaje de los úteros extirpados puede explicarse en base de que la indicación quirúrgica de histerectomía, tuvo muy en cuenta la 
edad de la paciente, practicando esta operación en las pacientes menopáusicas.

\section{CUADRO N: 15 \\ ESTUDIO HISTOLOGICO DE LOS UTEROS EXTIRPADOS}

\begin{tabular}{lrr}
\hline Entidad & N: de Casos & $\%$ \\
\hline Utero senil & 73 & 76.0 \\
Leiomiomas uterinos & 14 & 14.5 \\
Fibrosis uterina & 6 & 6.3 \\
Adenomiosis uterina & 3 & 3.2 \\
\cline { 2 - 2 } & 96 & $100 \%$ \\
\hline
\end{tabular}

\section{CUADRO № 16}

\section{HALLAZGOS HISTOLOGICOS EN 62 ESTUDIOS DE ENDOMETRIO, EN UTEROS OBTENIDOS POR HISTERECTOMIA VAGINAL, PARA TRATAMIENTO DEL PROLAPSO GENITAL}

\begin{tabular}{lcr}
\hline Endometrio & $N^{0}$ de Casos & $\%$ \\
\hline Atrófico & 27 & 43.5 \\
Folicular & 17 & 27.4 \\
Luteínico & 10 & 16.1 \\
Hiperestrogénico & 5 & 8.1 \\
Hiperplásico & 3 & 4.9 \\
\cline { 2 - 3 } & 62 & $100 \%$ \\
\hline
\end{tabular}

Puede observarse la cifra de mayor incidencia en el endometrio de tipo atrófico y muy escasa para los endometrios luteínicos, en vista de que la mayoría de las pacientes intervenidas para histerectomía vaginal, como tratamiento de su prolapso genital, se encontraban en grupos de edad avanzada.

Solamente se informaron dos quistes simples del ovario.

\section{RESUMEN}

Se analizan 478 intervenciones para Prolapso Genital Uterino, ingresadas al Departamento de Ginecología del Hospital Universitario San Vicente de Paúl en un período de abservación de 5 años (Enero 1960 Dic. 1964). Se destacan un alto porcentaje en la incidencia entre los 31 y 60 años, que alcanza el $75.4 \%$.

La mayor incidencia respecto a la paridad se encontró entre los cuatro y los 10 hijos con un porcentaje muy cercano al $60 \%$.

De acuerdo a la clasificación Internacional el III? de Prolapso ocupa el primer grado de frecuencia con el $50 \%$ de los casos.

Se analizan 436 estudios citológicos, según la técnica de Papanicolaou cuyos informes fueron 4 casos IV: $y$ seis $V:$. El análisis histopatológico de ellos se informó: para el grado IV un carcinoma intraepitelial y tres casos de metaplasia escamosa con atipias. Para los grados $V$ un carcinoma invasor y cuatro carcinomas intraepiteliales, se presentó también un caso de metaplasia escamosa con atipias.

En el período de observación analizádo hubo preferencia por la técnica de Mánchester (Donald Fothergill) con $46.2 \%$. La histerectomía vaginal y plastias complementarias ocupa el segundo lugar con $31.3 \%$.

Se establece una comparación en la morbilidad y complicaciones entre la operación de Mánchester y la Histerectomía Vaginal encontrando una incidencia mayor para la Histerectomía. La complicación más frecuente fue la infección urinaria.

Menos de la mitad de las pacientes asisten a la revisión post-quirúrgica, encontrando en ellas como secuela más frecuente la estrechez vaginal y dispareunia. 
Se reprodujo el Prolapso Integral en 7 casos: 3 después de una operación de Mánchester, dos después de plastias vaginales, uno después de la operación de Halban y una después de una fijación de Crossen. Recidivaron 8 cistoceles, 8 rectoceles, 5 colpoceles anterior $y$ posterior $y$ se presentaron 6 casos de prolapso de cúpula vaginal después de la histerectomía y plastias.

La anestesia utilizada fue: en el $66.5 \%$ de los casos general; en el $25.9 \%$ raquídea, en el $6.28 \%$ epidural y combinada en $1.26 \%$.

La cervicitis crónica y la cervicitis crónica con metaplasia escamosa constituyen el hallazgo del $80 \%$ de los cuellos extirpados.

El estudio Anatomopatológico de los úteros extirpados indicó la pre- sencia de un útero atrófico en el $76 \%$ de las piezas quirúrgicas.

\section{CONCLUSIONES}

1. El Prolapso genital continúa siendo en nuestro medio la primera ginecopatía de tipo quirúrgico en orden a la frecuencia.

2. La operación de Mánchester (Donald-Fothergill) predomina como técnica de elección en la corrección quirúrgica de todos los grados de prolapso independiente de la edad, paridad, y porvenir obstétrico (y sexual) de las pacientes.

3. No pueden evaluarse resultados definitivos debido al corto período de observación y al hecho de que las pacientes no asisten a la medida de nuestras ambiciones a revisiones quirúrgicas tardías. 